\title{
Herwiri2: CEEX Electroweak Corrections in a Hadronic MC
}

\author{
Scott Yost ${ }^{* \dagger}$ \\ The Citadel \\ Charleston, SC 29409, USA \\ E-mail: scott.yostecitadel.edu \\ Valerie Halyo \\ Princeton University \\ Princeton, NJ 08544, USA \\ E-mail: valerieh@princeton.edu
}

\section{Miroslav Hejna}

Princeton University

Princeton, NJ 08544, USA

E-mail: mhejna@princeton.edu

\section{B.F.L. Ward ${ }^{\S}$}

Baylor University Waco, TX 76798, USA

E-mail: bfl_ward@baylor.edu

\begin{abstract}
Reaching the $1 \%$ precision level for $W$ and $Z$ production calculations for the LHC will require a mixture of higher order QCD and electroweak corrections. As a first step toward implementing the combined QED $\otimes$ QCD exponentiation proposed in previous work, we have implemented the $\mathscr{O}(\alpha)$ electroweak corrections and YFS exponentiation structure of the $K K$ Monte Carlo in HERWIG. We discuss the current status of this program and sketch the further developments needed to reach the desired precision level.
\end{abstract}

10th International Symposium on Radiative Corrections (Applications of Quantum Field Theory to

Phenomenology)

September 26-30, 2011

Mamallapuram, India

\footnotetext{
* Speaker.

${ }^{\dagger}$ This work and its presentation were supported in part by D.o.E. grant DE-PS02-09ER09-01 and grants from The Citadel Foundation.

${ }^{\ddagger}$ Work supported in part by D.o.E grant DE-FG02-91ER40671.

${ }^{\S}$ Work supported in part by D.o.E. grant DE-FG02-09ER41600.
} 
Vector boson production is one of the most important Standard Model processes observed at the LHC, and electroweak radiative corrections will be needed for analysis at the percent level. Previous studies [1] by some of the authors have found that electroweak corrections alone can exceed $1 \%$ for some cuts of interest. These studies were based on HORACE, [2] which provides state-of-the-art $\mathscr{O}(\alpha)$ radiative corrections with a final-state photon shower, and PHOTOS, [3] which adds final state photonic radiation. Other programs developed for implementing electroweak corrections for hadronic collisions include WINHAC [4] and ZINHAC. [5] Initial state photonic radiation has been considered only in certain MRST parton distribution functions. [6] However, none of the most recent PDFs include QED effects.

For electron-positron colliders, precision electroweak corrections have been implemented in the program $K K \mathrm{MC}$ [7] using YFS [8] exponentiated multiple-photon radiation for both the initial and final state, together with $\mathscr{O}(\alpha)$ electroweak corrections in the DIZET [9] package developed for ZFITTER. [10] The DIZET corrections can be applied to any parton-level process, so there is no obstacle to extending them to hadronic collisions. YFS exponentiation provides the basis for an efficient representation of multi-photon phase space, which can also be implemented for hadronic initial and final states. Some of the authors have proposed using a non-abelian extension of YFS exponentiation as a basis for an integrated event generator implementing both multi-photon and multi-gluon corrections in a unified framework, called $\mathrm{QCD} \otimes \mathrm{QED}$ exponentiation. [11]

The collection of programs implementing $\mathrm{QCD} \otimes \mathrm{QED}$ exponentiation has been called HERWIRI, for High Energy Radiation with Infra-Red Improvements, with a version number distinguishing the class of corrections included. The name acknowledges that the initial versions build upon the HERWIG [12] parton shower generator. The first to be released, HERWIRI1, [13] implemented IR-improved splitting kernels [14] obtained using the QCD analog of YFS exponentiation. This program is publicly available, and tests are in progress. The IR-improved kernels have also been implemented [15] in MC@NLO [16] and POWHEG. [17] The structure of HERWIRI is not tied to a particular shower, and our ultimate goal is a complete shower generator based entirely on $\mathrm{QCD} \otimes \mathrm{QED}$ exponentiation with exact $\mathscr{O}\left(\alpha_{s}^{2}, \alpha_{s} \alpha, \alpha^{2}\right)$ residuals. [18]

The second version, HERWIRI2, implements the electroweak radiative corrections of $K K \mathrm{MC}$ in a hadronic shower generator. This note describes a version of HERWIRI2 which is presently nearing completion. ${ }^{1}$ HERWIRI2 is motivated by the successful application of YFS exponentiation in BHLUMI, [19] BHWIDE, [20], KoralZ, [21] KoralW, [22] YFSWW3, [23] KKMC, and related programs for LEP physics. All of these programs benefit from a very efficient representation of $N$ photon phase space, with complete control over the soft and collinear singularities for an arbitrary number of photons. Real and virtual IR singularities cancel exactly to all orders. The non-abelian extension to $\mathrm{QCD} \otimes \mathrm{QED}$ should have similar advantages.

While based on HERWIG, HERWIRI2 is largely independent of the underlying shower. HERWIG generates the parton momenta and shower, and HERWIRI2 passes the generated hard process momenta to $K K \mathrm{MC}$ routines to add photons and electroweak corrections. Although $K K \mathrm{MC}$ was developed for $e^{+} e^{-}$collisions, it was designed to be extended to more general processes, so the ability to select quarks as the incoming state already exists in all but the lowest-level generation routines.

\footnotetext{
${ }^{1}$ HERWIRI2 does not incorporate HERWIRI1, although the two programs can be used in combination.
} 
$K K \mathrm{MC}$ [7] is a precision generator for $e^{+} e^{-} \rightarrow f \bar{f}+n \gamma, f=\mu, \tau, d, u, s, c, b$ for CMS energies from $2 m_{\tau}$ to $1 \mathrm{TeV}$. The precision tag for LEP2 was $0.2 \%$. ISR and FSR $\gamma$ emission is calculated up to $\mathscr{O}\left(\alpha^{2}\right)$, including interference. The MC structure is based on YFS exponentiation, including residuals calculated perturbatively to the relevant orders in $\alpha^{k} L^{l}$. $\left(L=\ln \left(s / m_{e}^{2}\right)\right)$. Exact collinear bremsstrahlung is implemented for up to three photons. Electroweak corrections $[24,25,26]$ are included via DIZET 6.21, [9] and beamstrahlung can be modeled over a wide range of energies via a built-in or user-defined distribution.

There are two modes of operation: exclusive exponentiation (EEX) and coherent exclusive exponentiation (CEEX). EEX applies YFS exponentiation to differential cross-sections, while CEEX applies it at the amplitude level. The CEEX mode is written in a manner that is most readily extended to quark scattering, so it is taken as the basis for HERWIRI2. The orders of residuals included in CEEX mode are $\alpha, \alpha L, \alpha^{2} L^{2}$, and $\alpha^{2} L$.

CEEX was introduced for pragmatic reasons, because the traditional (EEX) exponentiation of spin-summed differential cross sections suffered from a proliferation of interference terms in processes with multiple diagrams, limiting its utility in reacing the desired $0.2 \%$ precision tag for LEP2.[27] CEEX works at the level of spinor helicity amplitudes, greatly facilitating the calculation of effects such as ISR-FSR interference, which are included in $K K \mathrm{MC}$, and therefore in HERWIRI2. CEEX is maximally inclusive: all real photons radiated are kept in the event record, no matter how soft or collinear. There is no need to integrate out a region of soft phase space, because the exponentiated amplitudes are well-behaved at $k=0$.

The CEEX cross section for $q \bar{q} \rightarrow f \bar{f}$ has the form [7]

$$
\sigma=\frac{1}{\text { flux }} \sum_{n=0}^{\infty} \int d \mathrm{PS} \rho_{\mathrm{CEEX}}^{(n)}(\vec{p}, \vec{k})
$$

where

$$
\rho_{\mathrm{CEEX}}^{(n)}=\frac{1}{n !} e^{Y\left(\vec{p}, E_{\min }\right)} \frac{1}{4} \sum_{\vec{\lambda}, \vec{\mu}}\left|\mathscr{M}\left(\begin{array}{cc}
\vec{p} & \vec{k} \\
\vec{\lambda} & \vec{\mu}
\end{array}\right)\right|^{2}
$$

The YFS form factor is

$$
\begin{aligned}
Y\left(\vec{p}, E_{\min }\right)= & Q_{i}^{2} Y\left(p_{1}, p_{2}, E_{\min }\right)+Q_{f}^{2} Y\left(p_{3}, p_{4}, E_{\min }\right)+Q_{i} Q_{f} Y\left(p_{1}, p_{3}, E_{\min }\right) \\
& +Q_{i} Q_{f} Y\left(p_{2}, p_{4}, E_{\min }\right)-Q_{i} Q_{f} Y\left(p_{1}, p_{4}, E_{\min }\right)-Q_{i} Q_{f} Y\left(p_{2}, p_{3}, E_{\min }\right),
\end{aligned}
$$

where $Q_{i}, Q_{f}$ are initial and final parton charges, and

$$
Y\left(p_{i}, p_{j}, E_{\min }\right)=2 \alpha \widetilde{B}\left(p_{i}, p_{j}, E_{\min }\right)+2 \alpha \operatorname{Re} B\left(p_{i}, p_{j}\right),
$$

with real and virtual form factors defined respectively by

$$
\begin{aligned}
& \widetilde{B}=-\int_{k^{0}<E_{\min }} \frac{d^{3} k}{8 \pi^{2} k^{0}}\left(\frac{p_{i}}{p_{i} \cdot k}-\frac{p_{j}}{p_{j} \cdot k}\right)^{2}, \\
& B=\frac{i}{(2 \pi)^{3}} \int \frac{d^{4} k}{k^{2}}\left(\frac{2 p_{i}+k}{2 p_{i} \cdot k+k^{2}}-\frac{2 p_{j}-k}{2 p_{j} \cdot k-k^{2}}\right) .
\end{aligned}
$$


The $n$-photon helicity-spinor amplitude can be expanded in terms of order $\alpha^{r}$ having the form

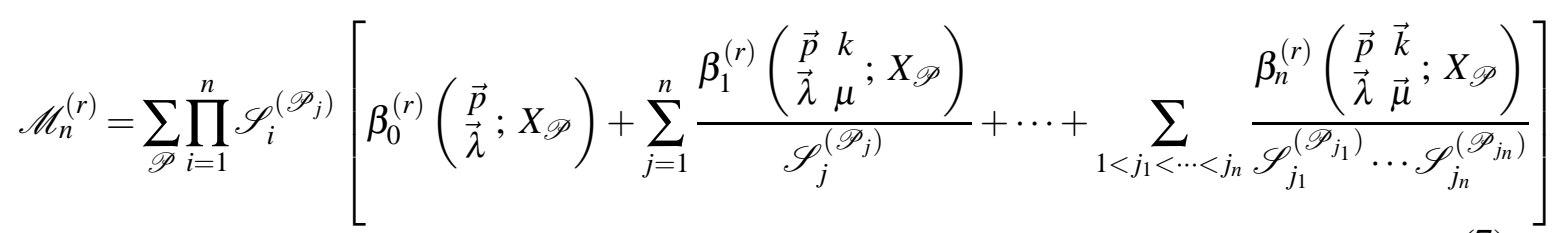

with residual spinor amplitudes $\beta_{i}^{(r)}$ and complex soft photon factors $\mathscr{S}_{j}$ with the property

$$
\left|\mathscr{S}_{j}^{\left(\mathscr{P}_{j}\right)}\right|=-2 \pi \alpha Q^{2}\left(\frac{p_{a}}{p_{a} \cdot k_{j}}-\frac{p_{b}}{p_{b} \cdot k_{j}}\right)^{2}
$$

where $Q, p_{a}, p_{b}$ belong to the initial or final fermions depending on the partition $\mathscr{P}_{j}$.

$K K \mathrm{MC}$ incorporates the DIZET library (version 6.21) from the program ZFITTER. [10] The $\gamma$ and $Z$ propagators are multiplied by vacuum polarization factors:

$$
H_{\gamma}=\frac{1}{2-\Pi_{\gamma}}, \quad H_{Z}=4 \sin ^{2}\left(2 \theta_{\mathrm{W}}\right) \frac{\rho_{\mathrm{EW}} G_{\mu} M_{Z}^{2}}{8 \pi \alpha \sqrt{2}}
$$

Vertex corrections are incorporated into the coupling of $Z$ to $f$ via form factors in the vector coupling:

$$
g_{V}^{(Z, f)}=\frac{T_{3}^{(f)}}{\sin \left(2 \theta_{W}\right)}-Q_{f} F_{\mathrm{v}}^{(f)}(s) \tan \theta_{W} .
$$

Box diagrams contain these plus a new angle-dependent form-factor in the doubly-vector component:

$$
g_{V}^{(Z, i)} g_{V}^{(Z, f)}=\frac{T_{3}^{(i)} T_{3}^{(f)}-2 T_{3}^{(i)} Q_{f} F_{\mathrm{v}}^{(f)}(s)-2 Q_{i} T_{3}^{(f)} F_{\mathrm{v}}^{(i)}(s)+4 Q_{i} Q_{f} F_{\mathrm{box}}^{(i, f)}(s, t)}{\sin ^{2}\left(2 \theta_{W}\right)} .
$$

The correction factors are calculated at the beginning of a run and stored in tables.

The Drell-Yan cross section with multiple-photon emission can be expressed as an integral over the parton-level process $q_{i}\left(p_{1}\right) \bar{q}_{i}\left(p_{2}\right) \rightarrow f\left(p_{3}\right) \bar{f}\left(p_{4}\right)+n \gamma(k)$, integrated over phase space and summed over photons. The parton momenta $p_{1}, p_{2}$ are generated using parton distribution functions giving a process at CMS energy $q$ and momentum fractions $x_{1}, x_{2}$ such that $q^{2}=x_{1} x_{2} s$ :

$$
\sigma_{\mathrm{DY}}=\int \frac{d x_{1}}{x_{1}} \frac{d x_{2}}{x_{2}} \sum_{i} f_{i}\left(q, x_{1}\right) f_{\bar{i}}\left(q, x_{2}\right) \sigma_{i}\left(q^{2}\right) \boldsymbol{\delta}\left(q^{2}-x_{1} x_{2} s\right),
$$

where the final state phase space includes $p_{3}, p_{4}$ and $k_{i}, i=1, \cdots, n$ and multiple gluon radiation + hadronization is included through a shower.

The parton-level cross section $\sigma_{i}\left(q^{2}\right)$ can be calculated by $K K \mathrm{MC}$, which integrates over a final space phase space with two fermions and an arbitrary number of photons:

$$
\sigma_{i}\left(q^{2}\right)=\sum_{n=0}^{\infty} \int d \mathrm{PS}_{2+n} \sigma_{i}(\vec{p}, \vec{k})
$$

HERWIRI2 uses HERWIG 6.5 as the shower generator, which creates the hard process first, at Born level, in subroutine HWEPRO (HWHDYP), and then passes it to the cascade generator HWBGEN. HERWIRI2 finds the $Z / \gamma^{*}$ and the partons interacting with it in the event record. The 
initial partons define $p_{1}, p_{2}$, which are transformed to the $\mathrm{CM}$ frame and projected on-shell to create a starting point for $K K \mathrm{MC}$, which generates the final fermion momenta $p_{3}, p_{4}$ and photons $k_{i}$ (both ISR and FSR.) The generated particles are transformed back to the lab frame and placed in the event record.

In addition to the basic DY process, HERWIG generates "Compton" events $g+q \rightarrow q+Z / \gamma^{*}$. About $10 \%$ of the events have this form. This is factorized into gluon emission times a hard EW process at a shifted value of $q^{2}$. These have a different profile in the event record, but can be processed by $K K \mathrm{MC}$ as well. There is also a third class of events with the emission of an additional hard gluon. About $1 \%$ of the events have this form, and also have a significant shift of the $Z$ energy from its generation scale.

With a change of variables, the Drell-Yan cross section in HERWIG can be expressed as

$$
\begin{aligned}
\sigma_{\mathrm{DY}} & =\int \frac{d x_{1}}{x_{1}} \frac{d x_{2}}{x_{2}} \sum_{i} f_{i}\left(q, x_{1}\right) f_{\bar{i}}\left(q, x_{2}\right) \sigma_{i}\left(q^{2}\right) \delta\left(q^{2}-x_{1} x_{2} s\right) \\
& =\int_{q_{\min }}^{q_{\max }} d q P(q) \int_{q^{2} / s}^{1} \frac{d x_{1}}{x_{1}} \sum_{i} P_{i} W_{\mathrm{HW}}^{(i)}\left(q^{2}, x_{1}\right)=\left\langle W_{\mathrm{HW}}\right\rangle
\end{aligned}
$$

where $P(q)$ is a normalized, integrable, crude probability distribution for $q, P_{i}$ is the crude probability of generating parton $i$, and $W_{\mathrm{HW}}$ is the HERWIG event weight. This weight depends only on the hard Born cross section and is not altered by the shower.

The crude probability distributions used by HERWIG are

$$
P(q)=\frac{1}{2}\left[P_{\gamma}(q)+P_{Z}(q)\right], \quad P_{\gamma}(q)=\frac{N_{\gamma}}{q^{4}}, \quad P_{Z}(q)=\frac{N_{2} q}{\left(q^{2}-M_{Z}^{2}\right)+\Gamma_{Z}^{2} M_{Z}^{2}}
$$

The HERWIG event weight is

$$
W_{\mathrm{HW}}=\sum_{i} W_{\mathrm{HW}}^{(i)}, \quad W_{\mathrm{HW}}^{(i)}=\frac{1}{P(q)} f_{i}\left(q, x_{1}\right) f_{\bar{i}}\left(q, x_{2}\right) \ln \left(\frac{s}{q^{2}}\right) \sigma_{\mathrm{HW}}^{(i)}\left(q^{2}\right)
$$

and the corresponding probability for selecting parton $i$ is

$$
P_{i}=W_{\mathrm{HW}}^{(i)} / W_{\mathrm{HW}}
$$

We have chosen to introduce electroweak corrections in a minimally invasive way, incorporating them in a form factor

$$
F_{E W}^{(i)}\left(q^{2}\right)=\frac{\sigma_{i}\left(q^{2}\right)}{\sigma_{\text {Born }}^{(i)}\left(q^{2}\right)}
$$

$K K \mathrm{MC}$ will calculate the EW form factor, and multiply it by the HERWIG Born cross section.

$$
\sigma_{\mathrm{HW}+\mathrm{EW}}=\left\langle W_{\mathrm{Tot}}\right\rangle, \quad W_{\mathrm{Tot}}=F_{E W}^{(i)}\left(q^{2}\right) W_{\mathrm{HW}}=W_{\mathrm{HW}} \frac{\sigma_{\mathrm{KK}}^{(i)}\left(q^{2}\right)}{\sigma_{\mathrm{Born}}^{(i)}\left(q^{2}\right)} .
$$

The $K K \mathrm{MC}$ cross section is calculated using a primary distribution

$$
\frac{d \sigma_{\mathrm{Pri}}^{(i)}(s, v)}{d v}=\sigma_{\mathrm{Born}}^{(i)}(s(1-v)) \frac{1}{2}\left(1+\frac{1}{\sqrt{1-v}}\right) \bar{\gamma}_{i} v^{\bar{\gamma}_{i}-1} v_{\min }^{\gamma_{i}-\bar{\gamma}_{i}}
$$


with

$$
\gamma_{i}=\frac{2 \alpha}{\pi} Q_{i}^{2}\left[\ln \left(\frac{s}{m_{i}^{2}}\right)-1\right], \quad \bar{\gamma}_{i}=\frac{2 \alpha}{\pi} Q_{i}^{2} \ln \left(\frac{s}{m_{i}^{2}}\right)
$$

to generate the factor $v$ giving the fraction of $s$ remaining after ISR photon emission, $s_{X}=s(1-v)$.

The $K K \mathrm{MC}$ cross section is

$$
\sigma\left(q^{2}\right)=\int d \sigma_{\mathrm{Pri}} \frac{d \sigma_{\mathrm{Cru}}}{d \sigma_{\mathrm{Pri}}} \frac{d \sigma_{\mathrm{Mod}}}{d \sigma_{\mathrm{Cru}}}=\sigma_{\mathrm{Pri}}\left\langle W_{\mathrm{Cru}} W_{\mathrm{Mod}}\right\rangle .
$$

$W_{\text {Cru }}$ is calculated during ISR generation and $W_{\text {Mod }}$ is generated after $s_{X}$ is available.

The HERWIG and $K K \mathrm{MC}$ weights are combined to calculate the total HERWIRI2 weight,

$$
\sigma_{\mathrm{Tot}}=\left\langle W_{\mathrm{HW}} \frac{\sigma_{i}\left(q^{2}\right)}{\sigma_{\mathrm{Born}}^{(i) \star}\left(q^{2}\right)}\right\rangle=\left\langle W_{\mathrm{HW}} \sigma_{\mathrm{Pri}}^{(i)}\left(q^{2}\right) \frac{W_{\mathrm{Cru}}^{(i)} W_{\mathrm{Mod}}^{(i)}}{\sigma_{\mathrm{Born}}^{(i) \star}\left(q^{2}\right)}\right\rangle,
$$

This average could be calculated using a joint probability distribution for $q$ and $v, D(q, v)=$ $P(q) d \sigma_{\mathrm{Pri}} / d v$, with $P(q)$ from HERWIG. An adaptive MC (S. Jadach's FOAM [28]) could calculate the normalization of the distribution at the beginning of the run, in a similar manner to how $K K \mathrm{MC}$ presently integrates the one-dimensional primary distribution. To account for beamsstrahlung, $K K \mathrm{MC}$ already permits such a user-defined distribution, in up to three variables. However, as a first step, we have tried to run HERWIRI2 using $K K \mathrm{MC}$ 's one-dimensional primary distribution. This requires fixing an overall scale $q_{0}$ to initialize $K K \mathrm{MC}\left(e . g ., q_{0}=M_{Z}\right)$.

The built-in primary distribution for electrons at scale $q_{0}$ can be used for the low-level generation of $v$. The transformation from this distribution to a distribution at HERWIG's generated scale $q$ for quark $i$ is then obtained by a change of variables:

$$
\sigma_{\mathrm{Tot}}=\sigma_{\mathrm{Pri}}^{(e)}\left\langle W_{\mathrm{HW}}\left(\frac{d \sigma_{\mathrm{Pri}}^{(i)}\left(q^{2}, v\right)}{d \sigma_{\mathrm{Pri}}^{(e)}\left(q_{0}^{2}, v\right)}\right)\left(\frac{W_{\mathrm{Crud}}^{(i)} W_{\mathrm{Mod}}^{(i)}}{\sigma_{\mathrm{Born}}^{(i) \star}\left(q^{2}\right)}\right)\right\rangle
$$

with

where

$$
\frac{d \sigma_{\mathrm{Pri}}^{(i)}\left(q^{2}, v\right)}{d \sigma_{\mathrm{Pri}}^{(e)}\left(q_{0}^{2}, v\right)}=W_{\gamma}^{(i)} \frac{\sigma_{\mathrm{Born}}^{(i)}\left(q^{2}(1-v)\right)}{\sigma_{\mathrm{Born}}^{(e)}\left(q_{0}^{2}(1-v)\right)}
$$

$$
W_{\gamma}=\frac{\bar{\gamma}_{i}}{\bar{\gamma}_{e}}\left(\frac{v}{v_{\min }}\right)^{\bar{\gamma}_{i}-\bar{\gamma}_{e}} v_{\min }^{\gamma_{i}-\gamma_{e}} .
$$

The $\gamma$ factors are calculated using $q^{2} / m_{i}^{2}$ for parton $i$ and $q_{0}^{2} / m_{e}^{2}$ for the electron.

Shuffling the numerators and denominators about gives the expression used in HERWIRI2:

$$
\sigma_{\mathrm{Tot}}=\left\langle W_{\mathrm{HW}} W_{\mathrm{Mod}} W_{\mathrm{Karl}} W_{\mathrm{FF}} W_{\gamma}\right\rangle
$$

with two new weights

$$
W_{\mathrm{Karl}}=\frac{\sigma_{\mathrm{Pri}}^{(e)} W_{\mathrm{Crud}}^{(i)}}{\sigma_{\mathrm{Born}}^{(e)}\left(q_{0}^{2}(1-v)\right)}, \quad W_{\mathrm{FF}}=\frac{\sigma_{\mathrm{Born}}^{(i)}\left(q^{2}(1-v)\right)}{\sigma_{\mathrm{Born}}^{(i) \star}\left(q^{2}\right)} .
$$

HERWIRI2 is still under development, so any numerical results must be treated as preliminary. A run for $p p$ collisions at $10 \mathrm{TeV}$ with the $Z / \gamma^{*}$ invariant mass bounded by $40 \mathrm{GeV}$ and 140 
GeV, using HERWIG 6.520 default parameters and PDFs, yields a cross-section of $1183.7 \pm 1.3$ $\mathrm{pb}$, compared to $1098.8 \pm 1.0 \mathrm{pb}$ for HERWIG alone, giving an electroweak contribution of of 7.7\%. Turning on ISR gives a much wider weight distribution and consequently, greatly reduced efficiency. Preliminary results give a cross-section of $1212 \pm 109 \mathrm{pb}$, showing an additional $2.4 \%$ contribution from ISR.

Work is in progress to optimize MC generation in the presence of ISR. Once HERWIRI2 is complete and thoroughly tested, it will be compared to other available hadronic/electroweak generators. In particular, it will be interesting to see the effect of initial state radiation, which is not present in the other programs, but appears to enter at the $2-3 \%$ level, making it crucial to precision calculations.

\section{Acknowledgments}

S. Yost thanks the organizers of RADCOR 2011 for the invitation to present these results, and D. Marlow and the Princeton Physics Department for their support and hospitality during a critical period of its development. S. Yost and B.F.L. Ward also acknowledge the hospitality of the CERN theory division, which contributed greatly to the progress of this work.

\section{References}

[1] N.E. Adam, V. Halyo, and S.A. Yost, JHEP 05 (2008) 062 [arXiv:0802.3251]; ibid., JHEP 11 (2010) 074 [arXiv:1006.3766]; N.E. Adam, V. Halyo, S.A. Yost, and W.-H. Zhu, JHEP 09 (2008) 133 [arXiv:0808.0758].

[2] C.M. Carloni Calame, G. Montagna, O. Nicrosini, and M. Treccani, JHEP 05 (2005) 019 [arXiv:hep-ph/0502218]; C.M. Carloni Calame, G. Montagna, O. Nicrosini, and A. Vicini, JHEP 12 (2006) 016 [arXiv:hep-ph/0609170]; ibid., JHEP 10 (2007) 109 [arXiv:0710.1722].

[3] E. Barberio, B. van Eijk, and Z. Wa̧s, Comput. Phys. Commun. 66 (1991) 115; E. Barberio and Z. Wa̧s, Comput. Phys. Commun. 79 (1994) 291; P. Golonka and Z. Wa̧s, Eur. Phys. J. C45 (2006) 97 [arXiv:hep-ph/0506026].

[4] W. Płaczek and S. Jadach, Eur. Phys. J. C29 (2003) 325 [arXiv:hep-ph/0302065]; W. Płaczek, PoS(EPS-HEP2009) 340 [arXiv:0911.0572].

[5] A. Siódmok and W. Płaczek, http://th-www.if.uj.edu.pl/ZINHAC/ .

[6] A.D. Martin, R.G. Roberts, W.J. Stirling, and R.S. Thorne, Eur. Phys. J. C39 (2005) 155.

[7] S. Jadach, B.F.L. Ward, and Z. Wa̧s, Comput. Phys. Commun. 130 (2000) 130.

[8] D.R. Yennie, S. Frautschi, and H. Suura, Ann. Phys. 13 (1961) 379.

[9] A. Akhundov, D. Bardin, M. Bilenky, P. Christova, S. Riemann, T. Riemann, M. Sachwitz, and H. Vogt, DIZET6.21

[10] A. Akhundov, D. Bardin, and T. Riemann, Phys. Lett. B166 (1986) 111.

[11] C. Glosser, S. Jadach, B.F.L. Ward, and S.A. Yost, Mod. Phys. Lett. A19 (2004) 2113 [arXiv:hep-ph/0404087]; B.F.L. Ward, C. Glosser, S. Jadach, and S.A. Yost, Int. J. Mod. Phys. A20 (2005) 3735 [arXiv:hep-ph/0411047]; B.F.L. Ward and S.A. Yost, in Proc. ICHEP04, Beijing, vol. 1, ed. H. Chen et al. (World Scientific, Singapore, 2005), 588 [arXiv:hep-ph/0410277]; ibid., Acta Phys. 
Polon. B38 (2007) 2395 [arXiv:0704.0294]; ibid., in Proc. ICHEP06, Moscow, vol. 1, 505 [arXiv:hep-ph/0610230]; ibid., PoS (RAD COR 2007) 038 [arXiv:0802.0724]; ibid.; B.F.L. Ward, S. Joseph, S. Majhi, and S.A. Yost, Proc. 2008 HERA-LHC Workshop, DESY-PROC-2009-02, eds. H. Jung, A. De Roeck (DESY, Hamburg, 2009) 180 [arXiv:0808.3133]; ibid., in Proc. ICHEP08, Philadelphia [arXiv:0810.0723].

[12] G. Corcella, I.G. Knowles, G. Marchesini, S. Moretti, K. Odagiri, P. Richardson, M.H. Seymour, and B.R. Webber, HERWIG6.5, arXiv:hep-ph/0011363.

[13] S. Joseph, S. Majhi, B.F.L. Ward and S.A. Yost, Phys. Lett. B685 (2010) 283 [arXiv:0906.0788]; ibid., Phys. Rev. D81 (2010) 076008 [arXiv:1001.1434]; ibid., Mod. Phys. Lett. A25 (2010) 2207; in Proc. DPF-2009, Detroit eConf C090726 [arXiv:0910.0491].

[14] B.F.L. Ward, Adv. High Energy Phys. 2008 (2008) 682312; Ann. Phys. 323 (2008) 2147.

[15] S. Joseph, S. Majhi, B.F.L. Ward and S.A. Yost, Pos (RADCOR2009) 070 [arXiv:1001.2730].

[16] S. Frixione and B. Webber, JHEP 0206 (2002) 029; S. Frixione, P. Nason, and B. Webber, JHEP 0308 (2003) 007.

[17] S. Alioli, P. Nason, C. Oleari, and E. Re, JHEP 0807 (2008) 060 [arXiv:0805.4802]; ibid., JHEP 1101 (2011) 095 [arXiv:1009.5594].

[18] S.A. Yost, Chris Glosser, and B.F.L. Ward, in Third International Symposium on Quantum Theory and Symmetries, Cincinnati, 2003 (World Scientific, Singapore, 2004) 775 [arXiv:hep-ph/0401211]; S.A. Yost and B.F.L. Ward, Nucl. Phys. (Proc. Supp.) B157 (2006) 78 [arXiv:hep-ph/0602030].

[19] S. Jadach, E. Richter-W as, B.F.L. Ward, and Z. Wa̧s, Comput. Phys. Commun. 70 (1992) 305; S. Jadach, W. Płaczek, E. Richter-W as, B.F.L. Ward, and Z. Wạs, Comput. Phys. Commun. 102 (1997) 229; B.F.L. Ward, S. Jadach, M. Melles, and S.A. Yost, Phys. Lett. B450 (1999) 262.

[20] S. Jadach, W. Płaczek, and B.F.L. Ward, Phys. Lett. B390 (1997) 298.

[21] S. Jadach, B.F.L. Ward, and Z. Wass, Comput. Phys. Commun. 79 (1994) 503.

[22] M. Skrzypek, S. Jadach, W. Płaczek, and Z. Wa̧s, Comput. Phys. Commun. 94 (1996) 216; S. Jadach, W. Płaczek, M. Skrzypek, B.F.L. Ward, and Z. Wạs, Comput. Phys. Commun. 119 (1999) 272.

[23] S. Jadach, W. Płaczek, M. Skryzpek, B.F.L. Ward, and Z. Wa̧s, Comput. Phys. Commun. 140 (2001) 432.

[24] D. Bardin, P. Christova, and O. Fedorenko, Nucl. Phys. B175 (1980) 435; Nucl. Phys. B197 (1982) 1; A. Akhundov, D. Bardin, and T. Riemann, Nucl. Phys. B276 (1986) 1; D. Bardin, S. Riemann, and T. Riemann, Z. Physik C32 (1986) 121; D. Bardin, S. Bilenky, G. Mitselmakher, T. Riemann, and M. Sachwitz, Z. Physik C44 (1989) 493; D. Bardin, W. Hollik, and T. Riemann, Z. Physik C49 (1991) 485 .

[25] A. Djouadi and C. Verzegnassi, Phys. Lett. B195 (1987) 265; A. Djouadi, Nuovo Cim. 100A (1988) 357.

[26] H. Burkhardt, F. Jegerlehner, G. Pensko, and C. Verzegnassi, Z. Physik C43 (1989) 497; F. Jegerlehner, in Progress in Particle and Nuclear Physics, Vol. 27, ed. A. Fassler (Pergamon Press, Oxford, 1991) 32.

[27] S. Jadach, B.F.L. Ward, and Z. Wa̧s, Phys. Rev. D63 (2001) 113009 [arXiv:hep-ph/0006359].

[28] S. Jadach, Comput. Phys. Commun. 130 (2000) 244 [arXiv:physics/99100004]. 